\title{
A multicenter cohort study of primary hypertension in Korea: study design and interim analysis of the Korean registry of target organ damage in hypertension (KorHR)
}

Suk-Won Choi ${ }^{1}$, Seong Woo Han ${ }^{1}$, Jong Sun Ok', Byung-Su Yoo ${ }^{2}$, Mi-Seung Shin ${ }^{3}$, Sung Ha Park ${ }^{4}$ and Kyu-Hyung Ryu ${ }^{1 *}$

\begin{abstract}
Background: The Korean Registry of Target Organ Damage in Hypertension aims to evaluate the clinical characteristics and prevalence of subclinical target organ damage in Korean hypertensive patients.

Method: This is a prospective, observational, multicenter cohort study in which 23 university hospitals participated. Since May 2013, we have enrolled 1,318 consecutive hypertensive patients without known cardiovascular disease who met the following inclusion criteria: 1) age older than 30 years and 2) the first visit to the participating hospitals was within the last 5 years.
\end{abstract}

Results: The mean age was $52 \pm 12$ years; $62.1 \%$ were male, and $41.3 \%$ were incident hypertensives. Patients with diabetes mellitus accounted for $7.8 \%$ of the population and $43.8 \%$ had hyperlipidemia or were on statins at baseline. The mean office blood pressures were 152 $\pm 20 / 96 \pm 14 \mathrm{mmHg}$ for incident hypertensive patients and 129 $\pm 13 / 78 \pm 10 \mathrm{mmHg}$ for patients on treatment. Patients with electrocardiographic and echocardiographic left ventricular hypertrophy accounted for 18.9 and $25.6 \%$, respectively. The mean brachial-ankle pulse wave velocity (PWV) was $1564 \pm 293 \mathrm{~m} / \mathrm{s}$ and $19.5 \%$ had PWV values of more than $1750 \mathrm{~cm} / \mathrm{s}$. Patients with microalbuminuria and chronic kidney disease accounted for 21 and 4\%, respectively. The first prescribed class of antihypertensive medications was angiotensin converting enzyme inhibitors in 2.9\%, angiotensin receptor blockers (ARBs) in 57.5\%, diuretics in $7.6 \%$, calcium channel blockers (CCBs) in $61.0 \%$, beta blockers in $17.3 \%$, and fixed dose combination pill in $27.8 \%$.

Conclusion: Our interim analysis shows that subclinical target organ damage in hypertension is considerably present for incident or treated hypertensive patients. CCBs and ARBs were the most commonly prescribed classes of antihypertensive medications and fixed dose combination pills were actively used in Korea.

Trial registration: NCT01861080. Registered 16 May 2013

Keywords: Target organ damage, Hypertension

\footnotetext{
* Correspondence: khryumd@hanmail.net

${ }^{1}$ Division of Cardiology, Department of Internal Medicine, Dongtan Sacred

Heart Hospital, Hallym University, 7 Keunjaebong-Gil, Hwaseong, Gyeonggido

18450 , South Korea

Full list of author information is available at the end of the article
} 


\section{Background}

Hypertension is an important worldwide public-health challenge because of its high frequency and concomitant risk of cardiovascular and kidney disease [1]. Substantial evidence has established that lowering high blood pressure (BP) is an effective way to prevent cardiovascular and renal diseases [2]. Along with controlling BP, identification and treatment of other cardiovascular risk factors, such as hyperlipidemia, smoking, and diabetes mellitus, is crucial in the management of hypertension.

Another important factors in the risk stratification and management of hypertensive patients are the existence of subclinical target organ damages (SODs) such as left ventricular hypertrophy (LVH), carotid wall thickening, increased pulsed wave velocity (PWV), chronic kidney disease (CKD) with a reduced estimated glomerular filtration rate (eGFR), and microalbuminuria (MAU) [3]. In the European hypertension guidelines, active search for SODs is highly recommended because it allows a better stratification of the cardiovascular risk and helps physicians select the most appropriate antihypertensive medicines [3].

Despite the emphasis of guidelines on active investigation for SOD, large-population based data are limited in terms of the prevalence of SODs in Korean hypertensives. Therefore, we aimed to determine the prevalence of SODs and distinct characteristics in Korean hypertensives by constructing a nationwide hypertension registry. Hereafter, we report the study design and interim analysis of the Korean Registry of Target Organ Damage in Hypertension (KorHR).

\section{Methods}

\section{Study design}

The KorHR is a prospective, observational, multicenter cohort study in which 24 university hospitals in the Republic of Korea participated. From May 2013, patients were consecutively enrolled if they 1 ) were older than 30 years old, 2) had primary hypertension, and 3) consented to registry enrollment. Study patients were classified into one of two tracks. One is a group of patients who were diagnosed with primary hypertension at enrollment and had never taken an antihypertensive medication (incident group). Patients were also considered as the incident group if they had been diagnosed with primary hypertension before enrollment and had not taken antihypertensive medications for more than 2 weeks from the time of enrollment. The second is a group of patients who had taken antihypertensive treatment before the study enrollment and first visited research institutions within the past 5 years (on-treatment group).

We excluded patients with obvious cardiovascular disease at baseline, such as cerebrovascular disease, coronary artery disease, heart failure, peripheral artery disease, and CKD with an eGFR of less than $30 \mathrm{~mL} / \mathrm{min} /$ $1.73 \mathrm{~m}^{2}$.

At the baseline visit, the demographic and anthropometric characteristics of eligible patients were recorded and the medical history, such as diabetes mellitus (DM), hyperlipidemia, regular exercise, and smoking were also reported. DM was defined as fasting plasma glucose $\geq$ $126 \mathrm{mg} / \mathrm{dL}$ or hemoglobin A1c $\geq 6.5 \%$ or if patient were taking anti-diabetic medications. Hyperlipidemia was defined as total cholesterol $\geq 240 \mathrm{mg} / \mathrm{dL}$ or low density lipoprotein $\geq 160 \mathrm{mg} / \mathrm{dL}$ or triglyceride $\geq 200 \mathrm{mg} / \mathrm{dL}$ or if patients were taking statins. Regular exercise was defined if patients do exercise (aerobic or anaerobic) for more than $30 \mathrm{~min}$ at a time and at least three times a week. The systolic blood pressure (SBP) and diastolic blood pressure (DBP) were measured two or three times in both arms at intervals of $3 \mathrm{~min}$ in accordance with the official recommendations for BP measurements [4]. The mean values of each measurement was recorded as the office BPs. Ambulatory BP monitoring was also used to rule out patients with white coat hypertension and evaluate the BP status. Laboratory tests were performed, including simple urinalysis, complete blood count, serum chemistry, serum lipid, serum uric acid, plasma renin activity, and aldosterone. The SODs of hypertension such as LVH, MAU, CKD, and increased PWV, were thoroughly scrutinized.

Finally, we investigated each class of antihypertensive medication that was prescribed first and analyzed a trend of antihypertensive treatment with medicines. We aimed to evaluate the cardiovascular outcomes for up to 5 years, including all cause death, cardiovascular death, ischemic or hemorrhagic stroke, myocardial infarction, coronary revascularization, and heart failure, although we do not report on the cardiovascular outcomes in this interim analysis.

\section{Definitions of the primary outcome}

The primary outcome was the presence of SODs of hypertension, such as $\mathrm{LVH}$, increased PWV, CKD with an eGFR of $30-60 \mathrm{~mL} / \mathrm{min} / 1.73 \mathrm{~m}^{2}$, and MAU. Electrocardiographic LVH was defined by the modified Sokolow-Lyon index (largest S-wave + largest R-wave $>$ $3.5 \mathrm{mV}$ ) and the assessment of echocardiographic LVH was based on the criteria proposed by the European hypertension guidelines [left ventricular mass index: men $>115 \mathrm{~g} / \mathrm{m}^{2}$ and women $>95 \mathrm{~g} / \mathrm{m}^{2}$ (BSA)] [3]. Left ventricular mass was calculated according to linear method using the following equation [5].

Left ventricular mass $=0.8 * 1.04 *$ [(thickness of interventricular septum at diastole + left ventricular internal diameter at diastole + posterior wall thickness at 
diastole $)^{3}-($ left ventricular internal dimension at diastole $\left.)^{3}\right]+0.6$ g.

The calculation of relative wall thickness (RWT) by the formula [(2*posterior wall thickness at diastole)/left ventricular internal dimension at diastole] permits the categorization of $\mathrm{LVH}$ as either concentric (RWT $>0.42$ ) or eccentric (RWT $\leq 0.42)$ [5]. MAU was defined as a urine albumin/creatinine ratio (ACR) in spot urine of $\geq 30 \mathrm{mg} / \mathrm{g}$ but $<300 \mathrm{mg} / \mathrm{g}$ [6]. Individuals with an eGFR $\leq$ $60 \mathrm{~mL} / \mathrm{min}$ per $1.73 \mathrm{~m}^{2}$ are defined as having CKD according to the K/DOQI clinical practice guidelines for CKD [7]. We calculated the eGFR using the Modification of Diet in Renal Disease Study equation [8]. The brachial-ankle pulse wave velocity (baPWV) was measured with the individuals in a supine position after at least $5 \mathrm{~min}$ of rest. Pressure waveforms of the brachial and tibial arteries were simultaneously recorded by placing occlusion cuffs connected to a plethysmographic sensor around both the brachia and ankles. The time delays $(T)$ of the two waveforms between one foot and the other were measured. The lengths of the paths from the suprasternal notch to the brachium (Lb) and from the suprasternal notch to the ankle (La) were automatically calculated according to the height of each individual. The baPWV was calculated using the following equation:

$$
\mathrm{baPWV}=\frac{(\mathrm{La}-\mathrm{Lb})}{T\left(\frac{m}{s}\right)}
$$

A threshold of $>1,750 \mathrm{~cm} / \mathrm{s}$ for the baPWV was determined for the presence of SOD, which was the cut-off value of baPWV for predicting cardiovascular disease in previous studies $[9,10]$.

\section{Statistical analysis}

Continuous variables were expressed as the mean \pm standard deviation (SD) and were compared using the $t$ test or Wilcoxon's rank-sum test. Categorical data were expressed as frequencies and percentages, and they were compared using the $\chi^{2}$ test or Fisher's exact test. Continuous variables were compared using the student's $t$ test or by the nonparametric Mann-Whitney $U$ test. For all tests, a probability value of $p<0.05$ was considered significant. All statistical analyses were performed using statistical software SPSS (Chicago, Illinois) version 19.0.

\section{Results}

As of April 2016, a total of 1,318 patients with primary hypertension had been enrolled in the KorHR registry. Of 1,318 eligible patients, 611 patients (46\%) were included in the incident group and the remaining $707 \mathrm{pa}-$ tients (54\%) were in the on-treatment group. Demographic and anthropometric characteristics of the patients are described in Table 1. In short, the mean age of all patients was 51 years and $60 \%$ of the patients were male. Patients with obesity (BMI $\geq 25 \mathrm{~kg} / \mathrm{m}^{2}$ ) accounted for $57.5 \%$. Hyperlipidemia (43.8\%) was the most common comorbidity. The mean overall office BP was $140 \pm$ $20 / 86 \pm 15 \mathrm{mmHg}$. Among patients in the on-treatments group, 534 (75.5\%) subjects had a BP that was lower than 140/90 mmHg. Table 2 shows the baseline laboratory data. Approximately $60 \%$ of study patients had impaired fasting glucose (fasting glucose more than $100 \mathrm{mg} / \mathrm{dL}$ but less than $126 \mathrm{mg} / \mathrm{dL}$ ). The average eGFR was $89 \pm 20 \mathrm{ml} / \mathrm{min} / 1.73 \mathrm{~m}^{2}$ (BSA) and mean LDL was $114 \pm 33 \mathrm{mg} / \mathrm{dl}$. The mean baPWV was $1558 \pm 285 \mathrm{~cm} / \mathrm{s}$ in all patients. In Fig. 1, 24-hour ambulatory BP monitoring is shown. Of 1,318 patients, 711 performed 24hour ambulatory BP monitoring. The mean 24-hour SBP and DBP were 138 and $91 \mathrm{mmHg}$, respectively.

The prevalence of SODs, which is the primary endpoint of this study, is shown in Fig. 2. Echocardiography was performed in 524 patients and 1,088 patients underwent electrocardiography. The prevalence of echocardiographic and electrocardiographic LVH were 25.6 and $18.9 \%$ in all patients, respectively. When comparing the incident group with the on-treatment group, although the overall echocardiographic LVH seemed to be more prevalent in the on-treatment group ( 28.8 vs. $22.8 \%, p=$ 0.132 ), it was not statistically significant (Table 3 ). In terms of hypertensive ventricular remodeling, eccentric LVH was significantly more frequent in the ontreatment group than the incident group (18.9 vs. $10.0 \%$, $p=0.004)$. However, electrocardiographic LVH was significantly more frequent in the incident group than the on-treatment group ( 22.8 vs. $14.9 \%, p=0.015)$. The test of MAU using ACR was performed in 940 patients. Among them, 179 patients (19.0\%) had MAU and 26 patients $(2.1 \%)$ had overt proteinuria. The value of the eGFR could be obtained in 1,161 patients and the prevalence of CKD, which was defined as an eGFR 30-60 ml/ $\min / 1.73 \mathrm{~m}^{2}$ (BSA), was $4 \%$. When we set the cut-off value of baPWV to $1,750 \mathrm{~m} / \mathrm{s}$, the prevalence of an increased PWV was $19.5 \%$.

The class of antihypertensive medicines prescribed at baseline was investigated. The most frequently prescribed class was calcium channel blockers (CCBs) (61\%), which was followed by angiotensin receptor blockers (ARBs) $(57.5 \%)$, beta blockers (17.6\%), diuretics (7.6\%), and angiotensin converting enzyme (ACE) inhibitors (2.9\%). Among them, $27.8 \%$ were prescribed with a fixed dose combination pill; most of these combination pills were an ARB plus CCB (83.6\%).

\section{Discussion}

This study shows the integrated prevalence of SODs for primary hypertension in hypertensive patients in Korea. 
Table 1 Baseline characteristics

\begin{tabular}{|c|c|c|c|}
\hline & Overall $(N=1,318)$ & Incident $(N=611)$ & On treatment $(N=707)$ \\
\hline Age, years $\pm S D$ & $52 \pm 12$ & $47 \pm 11$ & $56 \pm 11$ \\
\hline Males (\%) & $818(62.1)$ & $415(67.9)$ & $403(57.0)$ \\
\hline $\mathrm{BMI}^{\mathrm{a}}\left(\mathrm{kg} / \mathrm{m}^{2}\right) \pm \mathrm{SD}{ }^{\mathrm{b}}$ & $25.9 \pm 3.5$ & $26.2 \pm 3.6$ & $25.8 \pm 3.4$ \\
\hline Obesity $^{c}(\%)$ & 56.8 & 61.2 & 52.8 \\
\hline Waist $(\mathrm{cm}) \pm S D$ & $88 \pm 9$ & $89.7 \pm 8.8$ & $87 \pm 9$ \\
\hline Office SBP $(\mathrm{mmHg}) \pm S D$ & $140 \pm 20$ & $152 \pm 20$ & $129 \pm 13$ \\
\hline Office DBP $(\mathrm{mmHg}) \pm S D$ & $86 \pm 15$ & $96 \pm 14$ & $78 \pm 10$ \\
\hline Office HR (beats per minute) $\pm S D$ & $75 \pm 18$ & $77 \pm 12$ & $72 \pm 11$ \\
\hline Hypertension awareness (months) & $48 \pm 65$ & $22 \pm 40$ & $71 \pm 74$ \\
\hline Diabetes mellitus (\%) & 7.8 & 3.9 & 11.0 \\
\hline Hyperlipidemia (\%) & 43.8 & 35.1 & 51.0 \\
\hline Regular exercise (\%) & 32.3 & 28.8 & 39.8 \\
\hline \multicolumn{4}{|l|}{ Smoking (\%) } \\
\hline Never & 54.8 & 51.5 & 57.4 \\
\hline Past & 19.4 & 15.1 & 22.8 \\
\hline Current & 20.2 & 25.6 & 15.8 \\
\hline
\end{tabular}

${ }^{a} B M I$ body mass index; ${ }^{\mathrm{b}} S D$ standard deviation; ${ }^{\mathrm{C} O b e s i t y}$ defined as a $\mathrm{BMI} \geq 25 \mathrm{~kg} / \mathrm{m}^{2}$

SODs in primary hypertension were quite prevalent: 18.9\% for ECG LVH, 25.6\% for echo LVH, 19\% for MAU, and $19.5 \%$ for increased PWV.

With respect to the LVH, eccentric LVH (14.1\%) was slightly more frequent than concentric LVH (11.5\%) in all subjects. Notably, although concentric LVH seemed to be more common in the incident group than in the on-treatment group, albeit not statistically significant, eccentric LVH was significantly more frequent in the on-treatment group than in the incident group. This finding is consistent with previous studies, including a recent systemic review on echocardiographic LVH [11,

Table 2 Laboratory Data

\begin{tabular}{|c|c|c|c|}
\hline & Overall $(N=1,318)$ & Incident $(N=611)$ & On treatment $(N=707)$ \\
\hline Hemoglobin (g/dL) & $14.6 \pm 3.5$ & $14.9 \pm 1.6$ & $14.4 \pm 4.6$ \\
\hline Hematocrit (\%) & $43.0 \pm 4.5$ & $44.0 \pm 4.8$ & $42.1 \pm 3.9$ \\
\hline Glucose (mg/dL) & $108 \pm 25$ & $106 \pm 24$ & $109 \pm 26$ \\
\hline Impaired fasting glucose (\%) & 59.8 & 56.0 & 63.0 \\
\hline Total cholesterol (mg/dL) & $188 \pm 36$ & $200 \pm 36$ & $177 \pm 32$ \\
\hline Triglyceride (mg/dL) & $151 \pm 109$ & $166 \pm 128$ & $139 \pm 87$ \\
\hline $\mathrm{HDL}(\mathrm{mg} / \mathrm{dL})$ & $52 \pm 14$ & $51 \pm 15$ & $52 \pm 13$ \\
\hline $\mathrm{LDL}(\mathrm{mg} / \mathrm{dL})$ & $114 \pm 33$ & $127 \pm 32$ & $104 \pm 31$ \\
\hline Uric acid (mg/dL) & $5.5 \pm 2.4$ & $5.8 \pm 3.2$ & $5.3 \pm 1.4$ \\
\hline Estimated GFR $\left(\mathrm{mL} / \mathrm{min} / 1.73 \mathrm{~m}^{2}\right)$ & $89 \pm 20$ & $93 \pm 26$ & $87 \pm 21$ \\
\hline $\begin{array}{l}\text { Urine albumin creatinine ratio }(\mathrm{mg} / \mathrm{g}) \text {, } \\
\text { median ( } 1 / 4 \text { quartile, } 3 / 4 \text { quartile) }\end{array}$ & $10.3(4.9,24.8)$ & $10.5(5.0,23.7)$ & $10.1(4.8,25.5)$ \\
\hline Renin (ng/ml/h) & $3.35 \pm 6.75$ & $3.12 \pm 6.76$ & $4.16 \pm 6.71$ \\
\hline Aldosterone (pg/mL) & $9.8 \pm 7.5$ & $9.6 \pm 6.5$ & $10.42 \pm 10.05$ \\
\hline $\mathrm{PWV}^{\mathrm{a}}$ & $1558 \pm 285$ & $1569 \pm 289$ & $1543 \pm 278$ \\
\hline Central SBP & $133 \pm 20$ & $136 \pm 19$ & $130 \pm 21$ \\
\hline Central DBP & $88 \pm 13$ & $92 \pm 12$ & $83 \pm 12$ \\
\hline Augmentation index & $38 \pm 27$ & $33 \pm 24$ & $47 \pm 28$ \\
\hline
\end{tabular}

\footnotetext{
a PWV pulsed wave velocity
} 


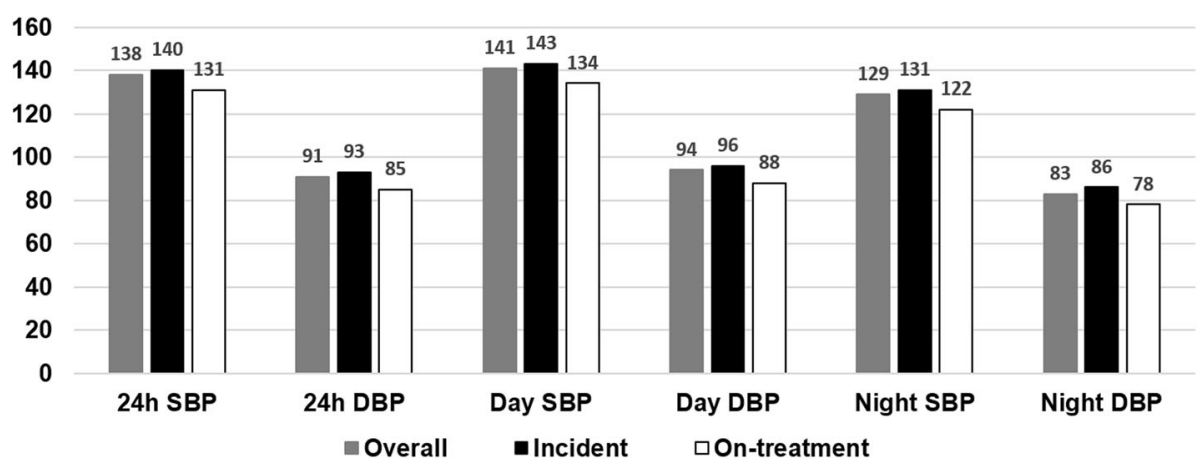

Fig. 1 Values of Twenty-four hour ambulatory blood pressure monitoring. 24 h, 24 h average; SBP, systolic blood pressure; DBP, diastolic blood pressure; Day, awake time average; Night, sleep time average

12]. Mancia et al. reported that eccentric LVH was more frequent than concentric $\mathrm{LVH}$ and the change in the left ventricular geometry due to high BP was retained regardless of whether the BP was normal or elevated and whether treatment did or did not achieve BP control [11]. As mentioned above, approximately $75 \%$ of patients in the on-treatment group in this study achieved target BP. As they proposed, the persistence of cardiac structure abnormalities such as LVH may not completely regress even after achieving target BPs. Additionally, the structural geometry may change with treatment, i.e., from concentric LVH to eccentric LVH with alteration of the RAS system. Concentric LVH was associated with a worse prognosis than the eccentric LVH [13]. However, eccentric LVH was also a significant predictor of the development of low LVEF. [14] Therefore, this result showed that even incident hypertensive patients are exposed to a risk of cardiovascular diseases in addition to patients on hypertensive treatment regardless of whether they achieved target BP. In contrast to echocardiographic LVH, electrocardiographic LVH was more prevalent in the incident group than in the on-treatment group. This finding can be explained that electrocardiographic change due to LVH and BP control might occur more easily than an echocardiographic left ventricular mass change.

MAU is known to be the integrated marker of SODs in primary hypertension [15]. MAU is associated with metabolic derangements and an increased cardiovascular risk in hypertension patients $[16,17]$. The previously reported prevalence of MAU varies from 4.7 to $40 \%$ according to study populations [18-23]. Among them, the I-DEMAND (Italy Developing Education and awareness on Microalbuminuria in hypertensive Disease) study, which enrolled patients with similar characteristics as this study, showed that the overall prevalence of MAU was $27 \%$ in 3,534 hypertensive patients [19]. Two previous Korean studies showed that the prevalence of MAU were 5.4 and $10.1 \%$, which included patients who visited primary clinics or participated in the health examination program [22, 23]. Several factors are known for affecting the prevalence of MAU. MAU is more frequent in

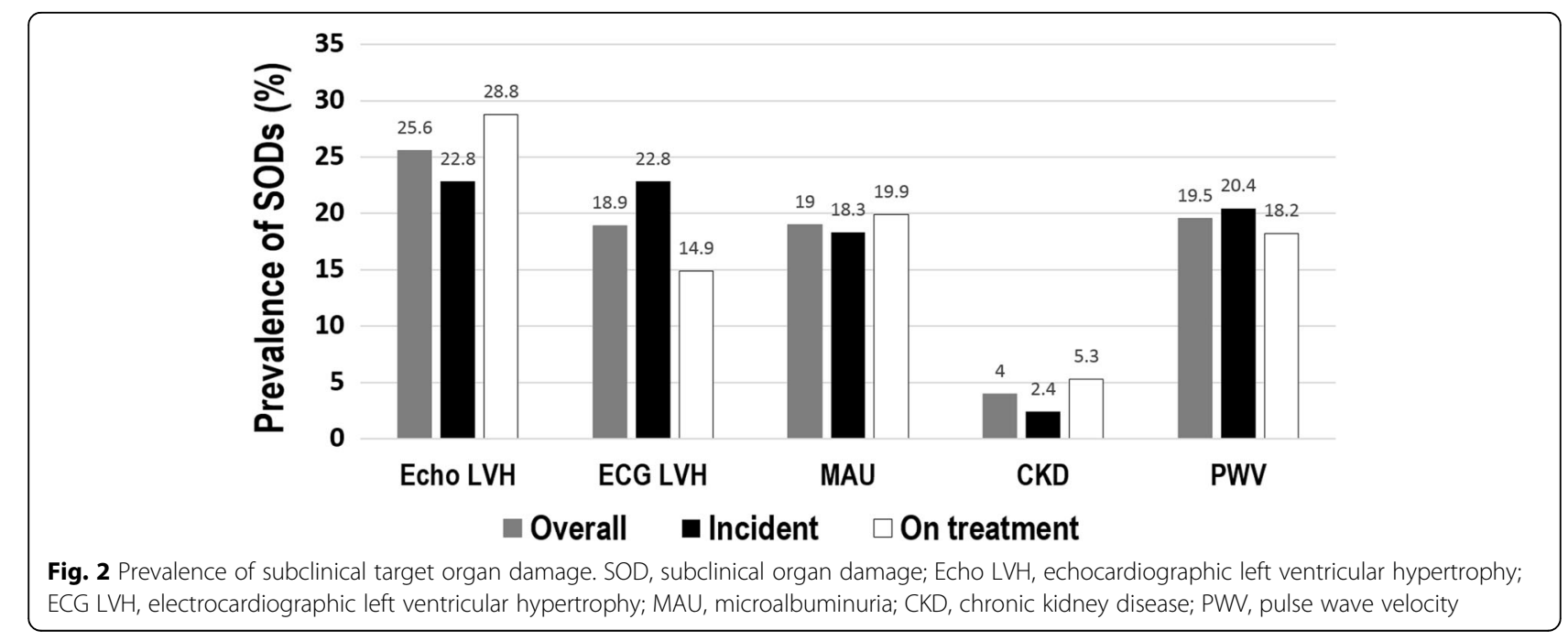


Table 3 Echocardiographic left ventricular hypertrophy

\begin{tabular}{lccrr}
\hline & Overall $(N=524)$ & Incident $(N=281)$ & On treatment $(N=243)$ & 28.8 \\
\hline LVH & 25.6 & 22.8 & 9.9 & 0.132 \\
Concentric LVH (\%) & 11.5 & 12.8 & 18.9 & 0.336 \\
Eccentric LVH (\%) & 14.1 & 10.0 & 12.3 & 0.004 \\
Concentric remodeling (\%) & 12.2 & 12.1 & 58.8 & 0.932 \\
Normal (\%) & 62.2 & 65.1 & 0.149 \\
\hline
\end{tabular}

patients with high stages of hypertension [24]. In addition to the severity of hypertension, age, sex, obesity, and concomitant risk factors could influence the presence of MAU, although other studies report conflicting results $[20,25-27]$. The difference between the present study and other Korean studies is mainly due to the characteristics of patients who visited different clinical settings, such as primary clinics and referral hypertension specialized clinics. The result suggests that MAU is significantly prevalent in Korean hypertensive patients and active investigation for MAU is encouraged for all hypertensive patients, as recommended by the guidelines. Although the prevalence of asymptomatic CKD was rare in incident hypertensives $(2.4 \%)$, it rose to a considerable level (5.3\%) in patients who have been treated with antihypertensive medicines for up to 5 years.

The carotid-femoral PWV (cfPWV) has been acknowledged as the standard of arterial stiffness [28]. However, the baPWV, which is much easier to measure, is more available in South Korea. Previous reports have shown that baPWV was correlated well with cfPWV and predicted worse outcomes in hypertensive patients $[29,30]$. These studies suggest that the baPWV cut-off value was approximately $1750 \mathrm{~cm} / \mathrm{s}$, which was associated with increased cardiovascular events and mortality $[9,10,31$, 32]. In this study, an overall prevalence of baPWV of more than $1750 \mathrm{~cm} / \mathrm{s}$ was $11.9 \%$. The prevalence of increased baPWV was significantly higher in the incident group than in the on-treatment group ( $16.0 \%$ vs. $8.3 \%, p$ $<0.001$ ), which could probably be affected by the high BP status of the incident group.

This study has limitations. First, we enrolled patients who visited hypertension specialized clinics in university hospitals. Because these patients could not be extrapolated to general population with hypertension, the prevalence of SODs could be exaggerated. Second, the performance rate of ECG, MAU, PWV, and 2D echocardiography was $82,71,61$ and, $40 \%$. Therefore, the findings of 2D echocardiography could be skewed. However, the prevalence of each SOD was quite comparable to previous studies.

Finally, our analysis of antihypertensive medicines demonstrated that CCBs and ARBs are the most commonly prescribed antihypertensive medicines. Notably,
ACE inhibitors were rarely prescribed, which is probably due to the high incidence of ACE inhibitor-induced cough in Asians [33]. Furthermore, fixed dose combination pills, which are expected to improve drug compliance, are actively used in South Korea. [34] In South Korea, there is no commercially available fixed dose combination pill that incorporates both an ACE inhibitor and $\mathrm{CCB}$, which could explain the high rate of $\mathrm{ARB}$ uses.

\section{Conclusions}

Interim analysis of the KorHR study showed that SODs of hypertension were prevalent in both incident and ontreatment hypertensive patients. In terms of medicines, CCBs and ARBs were the most commonly prescribed classes of antihypertensive medications and fixed dose combination pills are actively used in South Korea.

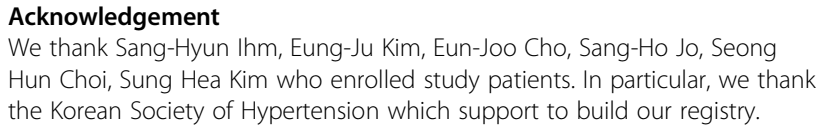

Availability of data and materials

Not applicable

\begin{abstract}
Authors' contribution
SWC and KHR recruited and analyzed patients' data and wrote the manuscript. JSO did data management. SWH, BSY, MSS, and SHP recruited enrolled study patients and contributed data analysis. All authors read and approved the final manuscript
\end{abstract}

\section{Competing interests}

The authors declare that they have no competing interests.

Ethics approval and consent to participate

The study was approved by the local ethics committee or institutional review board of each participating center. All patients provided written informed consent before enrollment.

Consent for publication

Not applicable

\section{Publisher's Note}

Springer Nature remains neutral with regard to jurisdictional claims in published maps and institutional affiliations. 


\section{Author details}

'Division of Cardiology, Department of Internal Medicine, Dongtan Sacred Heart Hospital, Hallym University, 7 Keunjaebong-Gil, Hwaseong, Gyeonggido 18450, South Korea. ${ }^{2}$ Division of Cardiology, Department of Internal Medicine, Yonsei University Wonju Severance Christian Hospital, Wonju, South Korea. ${ }^{3}$ Division of Cardiology, Department of Internal Medicine, Gachon University Gil Medical Center, Incheon, South Korea. ${ }^{4}$ Division of Cardiology, Department of Internal Medicine, Severance Cardiovascular Hospital, Seoul, South Korea.

\section{Received: 6 February 2017 Accepted: 12 June 2017}

\section{Published online: 02 August 2017}

\section{References}

1. Whelton PK. Epidemiology of hypertension. Lancet. 1994;344:101-6.

2. Czernichow S, Zanchetti A, Turnbull F, Barzi F, Ninomiya T, Kengne AP, et al. The effects of blood pressure reduction and of different blood pressurelowering regimens on major cardiovascular events according to baseline blood pressure: meta-analysis of randomized trials. J Hypertens. 2011;29:4-16.

3. Mancia G, Fagard R, Narkiewicz K, Redon J, Zanchetti A, Bohm M, et al. 2013 ESH/ESC guidelines for the management of arterial hypertension: the task force for the management of arterial hypertension of the European society of hypertension (ESH) and of the European society of cardiology (ESC). Eur Heart J. 2013:34:2159-219.

4. Pickering TG, Hall JE, Appel LJ, Falkner BE, Graves J, Hill MN, et al. Recommendations for blood pressure measurement in humans and experimental animals: part 1: blood pressure measurement in humans: a statement for professionals from the subcommittee of professional and public education of the American heart association council on high blood pressure research. Hypertension. 2005;45:142-61.

5. Lang RM, Bierig M, Devereux RB, Flachskampf FA, Foster E, Pellikka PA, et al. Recommendations for chamber quantification: a report from the American society of Echocardiography's guidelines and standards committee and the chamber quantification writing group, developed in conjunction with the European society of cardiology. J Am Soc Echocardiogr. 2005;18(12):1440-63.

6. Redon J. Urinary albumin excretion: lowering the threshold of risk in hypertension. Hypertension. 2005;46:19-20.

7. National Kidney Foundation. K/DOQI clinical practice guidelines for chronic kidney disease: evaluation, classification, and stratification. Am J Kidney Dis. 2002;39:S1-266.

8. Levey AS, Coresh J, Greene T, Stevens LA, Zhang YL, Hendriksen S, et al. Using standardized serum creatinine values in the modification of diet in renal disease study equation for estimating glomerular filtration rate. Ann Intern Med. 2006;145:247-54.

9. Kawai T, Ohishi M, Onishi M, Ito N, Takeya Y, Maekawa Y, et al. Cut-off value of brachial-ankle pulse wave velocity to predict cardiovascular disease in hypertensive patients: a cohort study. J Atheroscler Thromb. 2013;20:391-400

10. Munakata M, Konno S, Miura Y, Yoshinaga K. Prognostic significance of the brachial-ankle pulse wave velocity in patients with essential hypertension: final results of the J-TOPP study. Hypertens Res. 2012;35:839-42.

11. Mancia G, Carugo S, Grassi G, Lanzarotti A, Schiavina R, Cesana G, et al. Prevalence of left ventricular hypertrophy in hypertensive patients without and with blood pressure control: data from the PAMELA population. Pressioni Arteriose Monitorate E Loro Associazioni. Hypertension. 2002:39:744-9.

12. Cuspidi C, Sala C, Negri F, Mancia G, Morganti A. Prevalence of leftventricular hypertrophy in hypertension: an updated review of echocardiographic studies. J Hum Hypertens. 2012;26:343-9.

13. Muiesan ML, Salvetti M, Monteduro C, Bonzi B, Paini A, Viola S, et al. Left ventricular concentric geometry during treatment adversely affects cardiovascular prognosis in hypertensive patients. Hypertension. 2004;43:731-8,

14. Drazner MH, Rame JE, Marino EK, Gottdiener JS, Kitzman DW, Gardin JM, et al. Increased left ventricular mass is a risk factor for the development of a depressed left ventricular ejection fraction within five years: the cardiovascular health study. J Am Coll Cardiol. 2004:43:2207-15.

15. Leoncini G, Sacchi G, Ravera M, Viazzi F, Ratto E, Vettoretti S, et al. Microalbuminuria is an integrated marker of subclinical organ damage in primary hypertension. J Hum Hypertens. 2002;16:399-404.

16. Halimi JM, Forhan A, Balkau B, Novak M, Wilpart E, Tichet J, et al. Is microalbuminuria an integrated risk marker for cardiovascular disease and insulin resistance in both men and women? J Cardiovasc Risk. 2001;8:139-46.
17. de la Sierra A, Bragulat E, Sierra C, Gomez-Angelats E, Antonio MT, Aguilera MT, et al. Microalbuminuria in essential hypertension: clinical and biochemical profile. Br J Biomed Sci. 2000;57:287-91.

18. Calvino J, Calvo C, Romero R, Gude F, Sanchez-Guisande D. Atherosclerosis profile and microalbuminuria in essential hypertension. Am J Kidney Dis. 1999;34:996-1001.

19. Leoncini G, Viazzi F, Rosei EA, Ambrosioni E, Costa FV, Leonetti G, et al. Chronic kidney disease in hypertension under specialist care: the I-DEMAND study. J Hypertens. 2010;28:156-62.

20. Jensen JS, Feldt-Rasmussen B, Borch-Johnsen K, Clausen P, Appleyard M, Jensen G. Microalbuminuria and its relation to cardiovascular disease and risk factors. A population-based study of 1254 hypertensive individuals. J Hum Hypertens. 1997;11:727-32.

21. Cerasola G, Cottone S, Mule G, Nardi E, Mangano MT, Andronico G, et al. Microalbuminuria, renal dysfunction and cardiovascular complication in essential hypertension. J Hypertens. 1996;14:915-20.

22. Choi HS, Sung KC, Lee KB. The prevalence and risk factors of microalbuminuria in normoglycemic, normotensive adults. Clin Nephrol. 2006;65:256-61

23. Kim YS, Kim HS, Oh HY, Lee MK, Kim CH, Kim YS, et al. Prevalence of microalbuminuria and associated risk factors among adult Korean hypertensive patients in a primary care setting. Hypertens Res. 2013;36:807-23.

24. Jones CA, Francis ME, Eberhardt MS, Chavers B, Coresh J, Engelgau M, et al. Microalbuminuria in the US population: third National Health and Nutrition Examination Survey. Am J Kidney Dis. 2002;39:445-59.

25. Cirillo M, Senigalliesi L, Laurenzi M, Alfieri R, Stamler J, Stamler R, et al. Microalbuminuria in nondiabetic adults: relation of blood pressure, body mass index, plasma cholesterol levels, and smoking: the gubbio population study. Arch Intern Med. 1998;158:1933-9.

26. Pontremoli R, Sofia A, Ravera M, Nicolella C, Viazzi F, Tirotta A, et al. Prevalence and clinical correlates of microalbuminuria in essential hypertension: the MAGIC study. Microalbuminuria: a Genoa investigation on complications. Hypertension. 1997;30:1135-43.

27. Palatini P, Graniero GR, Mormino P, Mattarei M, Sanzuol F, Cignacco GB, et al. Prevalence and clinical correlates of microalbuminuria in stage I hypertension. Results from the hypertension and ambulatory recording Venetia study (HARVEST study). Am J Hypertens. 1996:9:334-41.

28. Laurent S, Cockcroft J, Van Bortel L, Boutouyrie P, Giannattasio C, Hayoz D, et al. Expert consensus document on arterial stiffness: methodological issues and clinical applications. Eur Heart J. 2006;27:2588-605.

29. Ninomiya T, Kojima I, Doi Y, Fukuhara M, Hirakawa Y, Hata J, et al. Brachialankle pulse wave velocity predicts the development of cardiovascular disease in a general Japanese population: the hisayama study. J Hypertens. 2013:31:477-83. discussion 483

30. Tanaka H, Munakata M, Kawano Y, Ohishi M, Shoji T, Sugawara J, et al. Comparison between carotid-femoral and brachial-ankle pulse wave velocity as measures of arterial stiffness. J Hypertens. 2009;27:2022-7.

31. Hae Guen S, Eung Ju K, Hong Seog S, Seong Hwan K, Chang Gyu P, Seong Woo $\mathrm{H}$, et al. Relative contributions of different cardiovascular risk factors to significant arterial stiffness. Int J Cardiol. 2010;139:263-8.

32. Sehestedt T, Jeppesen J, Hansen TW, Rasmussen S, Wachtell K, Ibsen H, et al. Thresholds for pulse wave velocity, urine albumin creatinine ratio and left ventricular mass index using SCORE, Framingham and ESH/ESC risk charts. J Hypertens. 2012;30:1928-36.

33. Watson RE, Karnchanasorn R, Gossain W. Hypertension in Asian/Pacific Island Americans. J Clin Hypertens (Greenwich). 2009;11:148-52.

34. Stanton T, Reid JL. Fixed dose combination therapy in the treatment of hypertension. J Hum Hypertens. 2002;16:75-8. 